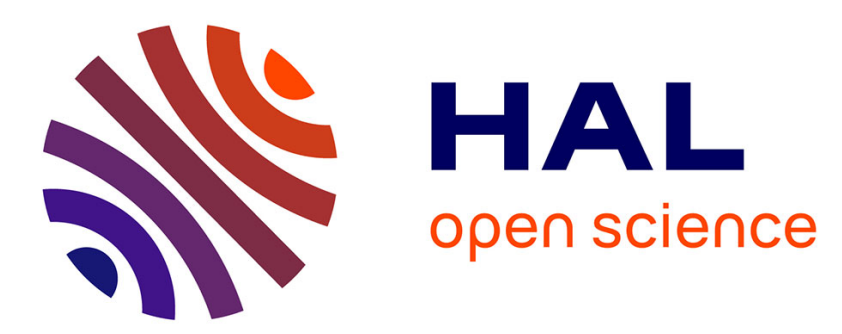

\title{
A Possible Nano-Scale Origin of the Surprising Thermal Expansion of Clays
}

\author{
Laurent Brochard, Túlio Honório, Matthieu Vandamme, Ioannis Stefanou,
} Siavash Ghabezloo, Michel Bornert

\section{- To cite this version:}

Laurent Brochard, Túlio Honório, Matthieu Vandamme, Ioannis Stefanou, Siavash Ghabezloo, et al.. A Possible Nano-Scale Origin of the Surprising Thermal Expansion of Clays. Sixth Biot Conference on Poromechanics, Jul 2017, Paris, France. pp.626, 10.1061/9780784480779.077 . hal-01686247

\section{HAL Id: hal-01686247 \\ https://hal.science/hal-01686247}

Submitted on 20 Jun 2018

HAL is a multi-disciplinary open access archive for the deposit and dissemination of scientific research documents, whether they are published or not. The documents may come from teaching and research institutions in France or abroad, or from public or private research centers.
L'archive ouverte pluridisciplinaire HAL, est destinée au dépôt et à la diffusion de documents scientifiques de niveau recherche, publiés ou non, émanant des établissements d'enseignement et de recherche français ou étrangers, des laboratoires publics ou privés. 


\title{
A possible nano-scale origin of the surprising thermal expansion of clays
}

\author{
L. Brochard ${ }^{1}$, T. Honorio ${ }^{2}$, M. Vandamme ${ }^{3}$, I. Stefanou ${ }^{4}$, S. Ghabezloo ${ }^{5}$ and M. \\ Bornert $^{6}$
}

${ }^{1}$ Laboratoire Navier, UMR 8205, Ecole des Ponts, IFSTTAR, CNRS, UPE, 6-8 avenue Blaise Pascal, 77455, Champs-sur-Marne, France; email: laurent.brochard@enpc.fr ${ }^{2}$ Laboratoire Navier, UMR 8205, Ecole des Ponts, IFSTTAR, CNRS, UPE, 6-8 avenue Blaise Pascal, 77455, Champs-sur-Marne, France; email: tulio.honorio-defaria@enpc.fr

${ }^{3}$ Laboratoire Navier, UMR 8205, Ecole des Ponts, IFSTTAR, CNRS, UPE, 6-8 avenue Blaise Pascal, 77455, Champs-sur-Marne, France; email: matthieu.vandamme@enpc.fr ${ }^{4}$ Laboratoire Navier, UMR 8205, Ecole des Ponts, IFSTTAR, CNRS, UPE, 6-8 avenue Blaise Pascal, 77455, Champs-sur-Marne, France; email: ioannis.stefanou@enpc.fr ${ }^{5}$ Laboratoire Navier, UMR 8205, Ecole des Ponts, IFSTTAR, CNRS, UPE, 6-8 avenue Blaise Pascal, 77455, Champs-sur-Marne, France; email: siavash.ghabezloo@enpc.fr ${ }^{6}$ Laboratoire Navier, UMR 8205, Ecole des Ponts, IFSTTAR, CNRS, UPE, 6-8 avenue Blaise Pascal, 77455, Champs-sur-Marne, France; email: michel.bornert@enpc.fr

\begin{abstract}
Clays thermo-mechanics are complex with thermal expansions or contractions, reversible or irreversible, depending on the loading history and the range of temperature. Some key experimental observations are well captured by a recent thermomechanical model considering water adsorption at the heart of the thermo-mechanical couplings. In this paper, we investigate a peculiar experiment that shows an effect of temperature on preconsolidation pressure. This experiment is interesting because it highlights a significant effect on the plastic behavior whereas the temperature change is applied after unloading in the elastic domain and induces only small reversible deformations. Accordingly, the phenomenon cannot be attributed to any change of microstructure. The model based on adsorption reproduces this experimental result and offers a nanoscopic interpretation of the effect of temperature on preconsolidation pressure. This finding further supports adsorption as central to the physical origin of the thermo-mechanical couplings in clays.
\end{abstract}

\section{INTRODUCTION}

Understanding the thermo-mechanical behavior of clays is important for a variety of applications from nuclear wastes storage to geothermal energy. This behavior is known to be quite uncommon, exhibiting thermal expansion or thermal contraction depending on the loading history of the material, with magnitudes much larger than 


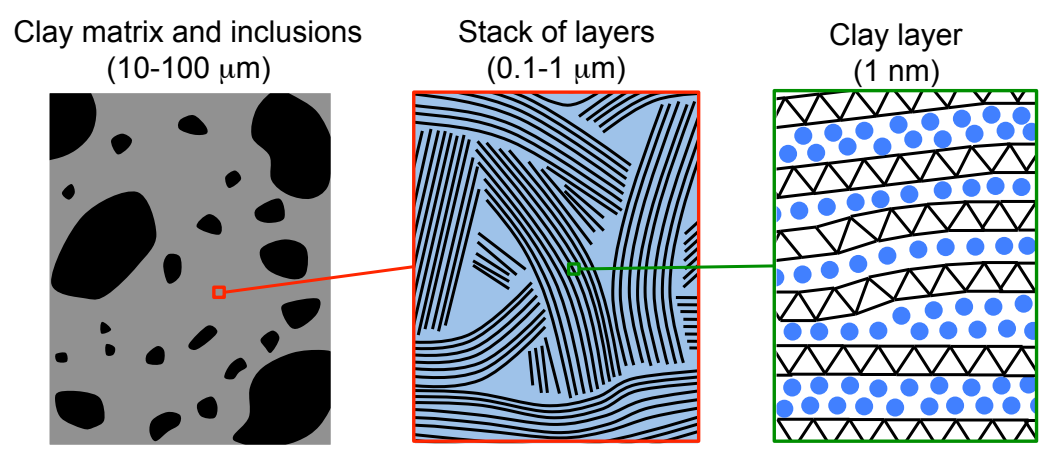

Figure 1. Schematic representation of the micro- and nanostructure of clays.

the thermal expansion of the solid minerals constitutive of clays. In the literature, this surprising behavior is usually attributed to the adsorbed water which is well known to induce volume changes upon desiccation or hydration. Yet, the physics of adsorption is generally disregarded in the geomechanical models. In a recent work, we proposed a new model of the thermo-mechanics of clays that does account for adsorption (Brochard et al., 2017). The models starts from the scale of a clay layer where water adsorption takes place up to the macroscopic scale of clay-rich soil and rock (Fig. 1). Using this model, we successfully capture the complex thermal expansion / contraction of a typical drained heating experiments and its dependency on the loading history. Moreover, the model is consistent with two other typical tests: consolidation at different temperatures and consolidation interrupted by heating-cooling cycles.

In this model, thermo-mechanical couplings are assumed to arise only from adsorption at the layer scale. Other possible phenomena may be at the origin of the couplings though, for instance the rearrangement of clay particles (stacks of layers). The typical tests considered so far show that adsorption could explain the thermo-mechanics of clays, but one may wonder whether adsorption is essential to a physical explanation of the thermo-mechanics of clays. To investigate this question, in this paper, we focus on another experiment presented in Figure 2. This experiment, from Habibagahi (1977), consists in a consolidation test during which the temperature is changed. The temperature change is performed after unloading the material. Doing so, the material is over-consolidated and its response to temperature change is small and reversible. When loading is resumed, one observes that the pre-consolidation pressure (pressure when plastic behavior is recovered) has changed. It has increased upon cooling and decreased upon heating.

In the next section, we recall briefly the thermo-mechanical model based on adsorption (Brochard et al., 2017). Then, we apply this model to the test of Habibagahi (1977), and we discuss the results. 

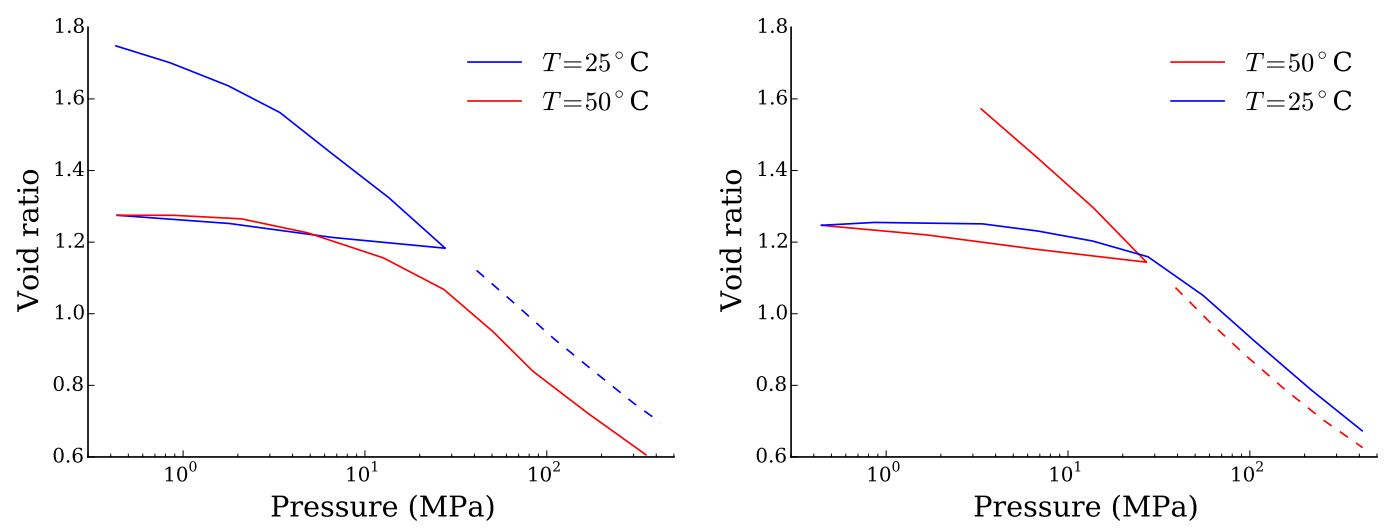

Figure 2. Consolidation test on clay interupted by temperature changes after unloading, adapted from Habibagahi (1977). This result proves that temperature has an impact on preconsolidation pressure, even if no irreversibility occurs during the change of temperature. (left) Case of an increase of temperature: the preconsolidation pressure decreases. (right) Case of a decrease of temperature: the preconsolidation pressure increases.

\section{MODEL OF THE THERMO-MECHANICAL BEHAVIOR OF CLAYS}

Let us first recall the main aspects of the thermo-mechanical model (for a more complete description, cf. Brochard et al. (2017)). The model starts by considering the effect of adsorption at the layer scale. Because of adsorption, the confining pressure at the layer scale oscillates with the basal spacing (Fig. 3). As a consequence, under controlled confining pressure, some basal spacings are 'authorized' or (meta)-stable (decreasing branches) and others are 'forbidden' or unstable (increasing branches). The physical origin of this behavior is the structuration of the adsorbed water: each range of 'authorized' spacings corresponds to an integer number of water layers (integer should be interpreted as an average since fluctuations of thermodynamic quantities are inherent at this scale). The confining pressure isotherm displayed in Figure 3 is obtained by molecular simulation (Grand Canonical ensemble) of a drained 2D toy model and exhibits many oscillations. For real clays in geological conditions (i.e., subjected to confining stress), the number of water layers is typically comprised between 0 and 3 (Meunier, 2005). The results of our work holds as long as at least one oscillation is present in the confining pressure isotherm (which is the case for swelling clays).

At the scale of a clay particle (stack of clay layers), several hydration states (number of water layers) or 'phases' can coexist. The situation of a particle is analogous to that of shape memory alloys (SMA) in which, because of very peculiar crystallography, several crystal phases can coexist within the microstructure (Bhattacharya, 2003). We build on the well-established theory of SMA to propose a mechanical description of a stack. At equilibrium, the mechanics of a stack follows the convex envelop of 

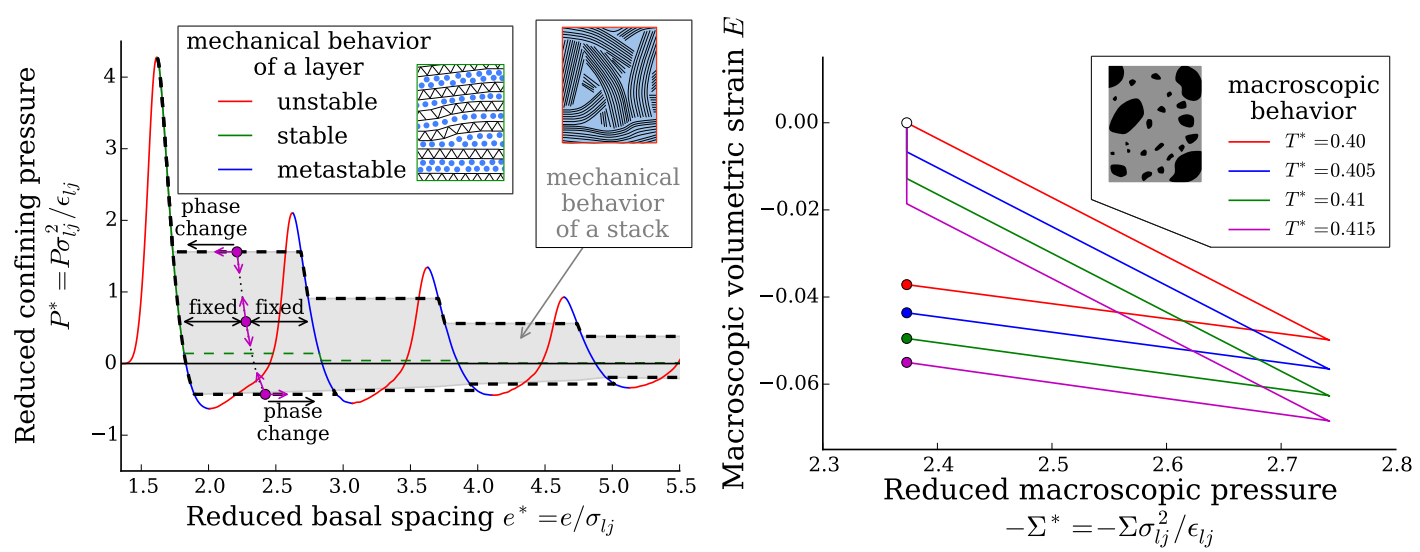

Figure 3. The thermo-mechanical model considers three scales. At the scale of a layer the confining stress is an oscillating function of the basal spacing. Each (meta)-stable branch is a phase corresponding to an integer number of water layers between the solid minerals. At the scale of a particle (stack of layers), different phases can coexist. The mechanics of a stack thus follows the convex envelop of the free energy (stable). In presence of meta-stability, the mechanical behavior of a stack occupies a 'domain' in which phase transitions are possible only along the horizontal boundaries. Finally, the macroscopic behavior is derived from the particle behavior by mean of self-consistent homogenization. All reported quantities are reduced in dimensionless form with respect to the Lennard-Jones parameters $\sigma_{i j}$ and $\epsilon_{i j}$ corresponding to the molecular interactions within the adsorbed fluid (cf. Brochard et al., 2017). 
the free energy of a layer. In non-convex portions, the convex envelop follows straight lines, corresponding to constant confining pressure, along which phase transition can occur. This provides the stable behavior in green in Figure 3. The dashed parts are the phase transitions. Alongside the stable behavior, one can observe meta-stable states. Estimates of the energy barriers to overcome meta-stability are generally much larger than thermal agitation, thus prone to metastability. Metastability is accounted for in the mechanical behavior of a stack by considering phase transitions at pressures different from the stable case. In our model, the pressures of phase transitions were chosen to satisfy a given ratio between energy barrier and thermal agitation energy. In fine, one obtains the grey domain in Figure 3 for the mechanical behavior of a stack. Phase transitions are possible at the horizontal boundaries of the domain only. The mechanical behavior of the layer and particle depends on temperature. In particular the amplitude of the confining pressure oscillations are reduced upon heating, and so is the corresponding phase transition pressure for the particle.

Finally, the macroscopic behavior at the scale of the clay matrix and inclusions is obtained through self-consistent homogenization. We consider an incremental formulation with pre-stresses to account for non linearities and thermal expansion / contraction. The microstructure of the material is made of $50 \%$ of anisotropic inclusions representing the clay particles, and 50\% of isotropic linear elastic inclusions representing the other minerals in clay rocks (e.g., carbonates, silica etc.). In the present implementation of the model, all clay particles are in equal proportions (isotropic crystallographic texture) and constitutive phases are represented in the self-consistent approach as spherical Eshelby-type inclusions, which corresponds an isotropic morphological texture. Under such conditions, the response of the material to the purely spherical loading conditions considered here is spherical, and can be characterized by pressure versus volume change curves, parameterized by temperature. In Figure 3, we illustrate the macroscopic behavior with the example of the response to consolidation at different temperatures. Temperature is known to translate the consolidation curve to lower strains with no impact on the slopes (plastic and elastic compressibilities), which is well reproduced by our model.

\section{APPLICATION TO THE EXPERIMENT OF HABIBAGAHI (1977)}

In this section, we apply to our model a loading reproducing the conditions of the experiment of Habibagahi (1977) (cf. Fig. 2). We start from a normally consolidated material. A normally consolidated clay is a clay whose current macroscopic confining pressure is the highest it has experienced in its geological history. A way to achieved normal consolidation is to consider remolded clay, which is supposed to relax the internal stresses, and consolidate the material to the confining pressure of interest. Here, we follow this principle to generate a normally consolidated state (cf. Brochard et al. 2017 for more details). We first initialize the material with particle inclusions at large basal spacings and small confining pressure, and the same confining pressure was applied to 
all the inclusions in all directions. We then consolidate the material by increasing the macroscopic confining pressure to reach small basal spacings (here between the second and third oscillations). Starting from this normally consolidated state, we perform a loading corresponding to the experiment of Habibagahi (1977):

1. We consolidate further the material by increasing the macroscopic confining pressure.

2. We unload the material by decreasing the macroscopic confining pressure.

3. We change the temperature. Two cases are considered, the first one with temperature increase and the second with temperature decrease.

4. We resume the consolidation by increasing the macroscopic confining pressure.

The macroscopic and microscopic responses of the material are displayed in Figure 4. The macroscopic response is consistent with the experimental observations of Habibagahi (1977) (Fig. 2): the change of temperature is small and reversible and the preconsolidation pressure is modified after the temperature change. A temperature increase decreases the preconsolidation pressure, and conversely a temperature decrease increases the preconsolidation pressure. The model offers an interpretation of this phenomenon at the layer scale: during a plastic consolidation, phase transitions occur within a clay particle and the state of a particle moves along the horizontal line corresponding to the phase transition pressure. When the material is unloaded, the state of the clay particle lies below the phase transition pressure and no more phase change is possible. At this point the material is over consolidated and the change of temperature leads to a small reversible deformations. However, even if the behavior remained in the elastic domain, the phase transition pressure, i.e., the boundary of the domain, has been modified by the temperature change. Since the amplitude of the confining pressure oscillations at the layer scale decreases upon temperature increase, so does the phase transition pressure. Thus, when consolidation is resumed, the plastic regime is recovered at a different loading as before the temperature change: for temperature increase, the phase transition pressure is smaller and the plastic regime is recovered at a lower loading, that is the preconsolidation pressure has decreased; for a temperature decrease, the phase transition pressure is larger and the plastic regime is recovered at a larger loading, that is the preconsolidation pressure has increased.

What makes this test particularly interesting is the fact that the plastic behavior is affected while the change of temperature induces only negligible deformations with no irreversibility. Our model offers a possible nanoscale explanation to this observation. In our model, clay plasticity arises from the phase changes within the particle. Another likely physical origin of plasticity is the sliding of clay layers within a particle, which is also strongly related to water adsorption and similar effect of temperature could be expected. Disregarding adsorption, one could explain plasticity from a purely 

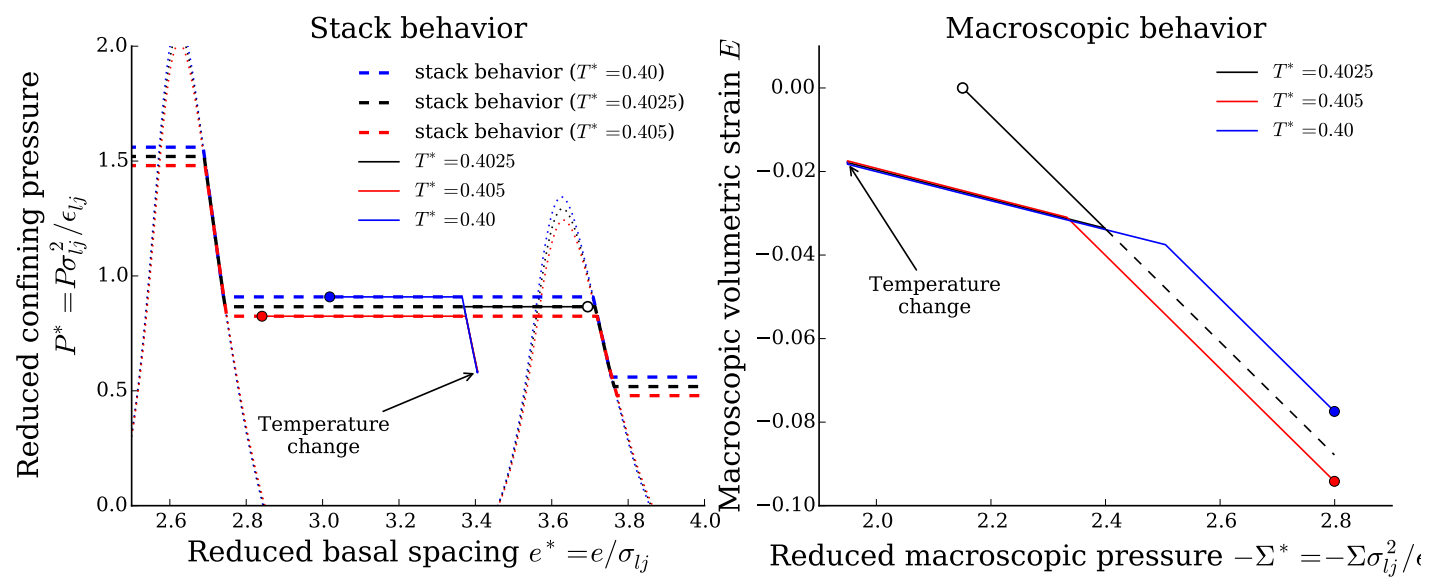

Figure 4. Mechanical response of the model to a loading reproducing the test of Habibagahi (1977). (left) Mechanical response at the stack scale. (right) Mechanical response at the macroscopic scale. Reported quantities are reduced as in Figure 3.

granular point of view (see for instance Sibille et al. (2015)). Based on such description, one could expect a change of preconsolidation pressure if the microstructure (arrangement of clay particles) or the inter-particle contact law have been modified during the temperature change. Microstructure changes are very limited since temperature change induces only small reversible deformations. Accordingly, a change in the contact law seems more plausible to explain the experiment from a granular perspective. How the contact law depends on temperature may well involve adsorption as clay particle surfaces are also subject to adsorption.

\section{CONCLUSION}

In this paper, we investigate the physical origin of the complex thermomechanics of clays. More specifically, we study the experiment of Habibagahi (1977) which provides evidence of an effect of temperature on preconsolidation pressure. We find that a thermo-mechanical model we developed in a previous work well captures this phenomenon. In this model, adsorption at the scale of the clay layers is the main physical origin of the thermo-mechanical couplings. This model offers a possible nanoscale interpretation of the effect of temperature on preconsolidation pressure: as temperature increases, maximum adsorption-induced pressure within the clay layers is reduced thus leading to a decrease of preconsolidation pressure. Interestingly, in the experiment of Habibagahi (1977), plasticity is affected by temperature whereas the temperature change is applied to an over consolidated state and induces only small reversible deformations. Therefore, the effect on plasticity is unlikely to be the consequence of a change of microstructure. The theory we propose here offers a possible physical explanation based on the clay layer behavior. 


\section{ACKNOWLEDGMENT}

We gratefully acknowledge funding through the project TEAM2ClayDesicc from the French National Research Agency (Agence Nationale de la Recherche, contract ANR-14-CE05-0023-01).

\section{REFERENCES}

Bhattacharya, K. (2003). Microstructure of martensite: why it forms and how it gives rise to the shape-memory effect. Oxford University Press.

Brochard, L., Honòrio, T., Vandamme, M., Bornert, M., and Peigney, M. (2017). "Nanoscale origin of the thermo-mechanical behavior of clays." In Preparation.

Habibagahi, K. (1977). "Temperature effect and the concept of effective void ratio." Indian Geotechnical Journal, 7(1), 1434.

Meunier, A. (2005). Clays. Berlin/Heidelberg: Springer-Verlag.

Sibille, L., Hadda, N., Nicot, F., Tordesillas, A., and Darve, F. (2015). "Granular plasticity, a contribution from discrete mechanics." Journal of the Mechanics and Physics of Solids, 75, 119139. 